\title{
Satisfação, confiança e lealdade em clientes de E-service: uma análise de fatores de influência em sites de notícias
}

Stephanie Ingrid Souza Barboza

Graduada em Administração (UFCG), doutora em Administração (UFPB), professora do Departamento de Ciências Sociais Aplicadas da UFPB/Campus III. Pesquisa na área de Marketing, Paraíba, Brasil

stephanieisb@gmail.com

Diana Lúcia Teixeira de Carvalho

Graduada em Publicidade e Propaganda (IESP), doutora em Administração (UFPB), professora do Departamento de Administração da UFPB/Campus I. Pesquisa na área de Marketing, Paraíba, Brasil

dinda_carvalho@hotmail.com

Francisco José da Costa

Graduado em Administração (UECE) e Estatística (UFPB), doutor em Administração (FGV/SP), professor do Departamento de Administração da UFPB/Campus I.

Pesquisa nas áreas de Marketing e Métodos quantitativos, Paraíba, Brasil

franze.mq@gmail.com

Editor Científico: José Edson Lara

Organização Comitê Científico

Double Blind Review pelo SEER/OJS

Recebido em 09.07.2016

Aprovado em 28.06.2017 


\title{
Resumo
}

O objetivo do artigo é analisar, sob uma perspectiva integrada e simultânea, a relação das características de inovação e qualidade de sites de notícias com a confiança, a satisfação e a lealdade do cliente usuário. A partir de uma revisão teórica, um modelo de hipóteses foi elaborado para teste em um estudo empírico. Foi feito o levantamento de campo através de um questionário estruturado, disponibilizado na internet, e que resultou em uma amostra de duzentos e trinta e nove respondentes. $\mathrm{Na}$ análise, foram empregadas a avaliação de consistência psicométrica e modelagem de equações estruturais com estimação por mínimos quadrados parciais. Como resultados, verificou-se principalmente que há uma influência da inovação e da qualidade do site na satisfação e na confiança dos usuários de sites de notícias, enquanto que a relação da lealdade ocorreu apenas com a satisfação, não havendo influência do nível de confiança do cliente usuário em sua lealdade.

Palavras-chave: E-service; Sites de notícias; Satisfação; Confiança; Lealdade

\section{Satisfaction, trust and loyalty in E-service customers: an analysis of factors influencing news sites}

\begin{abstract}
The aim of this article is to analyze, under an integrated and simultaneous perspective, the relation of the characteristics of innovation and quality of news websites with the trust, satisfaction and loyalty of the users. From a theorical review, a model of hypotheses was elaborated, to be tested in a empiric study. A field survey was made using a structured questionnaire, available on the Internet, and which resulted in a sample of two hundred thirty-nine respondents. At the analysis, were used evaluation of the psychometric consistency and structural equation model with estimation of partial least squares. As results, it was verified mainly that there is influence of the sites' innovation e quality on the satisfaction and trust of news websites users, meanwhile the relation of trust occurred only with satisfaction, there is no influence of the users's level of trust on their loyalty.
\end{abstract}

Key-Words: E-service; News websites; Satisfaction, Trust; Loyalty

\section{Satisfacción, confianza y lealtad en clientes de E-service: un análisis de factores de influencia en sitios de noticias}

\section{Resumen}

El objetivo de este trabajo es analizar, en una perspectiva integrada y simultánea, la relación de las características de innovación y calidad de los sitios de noticias con la confianza, la satisfacción y la lealtad de los usuarios. Después de una revisión 
teórica, se elaboró un modelo de hipótesis, para ser testado en un estudio empírico. Una encuesta fue realizada utilizando un cuestionario estructurado, disponible en Internet, que resultó en una muestra de doscientos treinta y nueve personas. En el análisis, se utilizó la evaluación de consistencia psicométrica y de modelos de ecuaciones estructurales con estimación de mínimos cuadrados parciales. Como resultados, se verificó principalmente que existe influencia de la innovación e calidad de los sitios de noticias en la satisfacción y confianza de los usuarios, mientras que la relación de confianza se produce sólo con satisfacción, no habiendo influencia del nivel de confianza de los usuarios en su lealtad.

Palabras clave: E-servicio; Sitios web de noticias; Satisfacción, Confianza; Lealtad

\section{Introdução}

Este estudo analisa o comportamento do consumidor usuário de serviços de sites de notícias. Esses tipos de serviços são recebidos via internet, por meio do acesso pelos usuários de informações, matérias e notícias diversas. No Brasil, são exemplos de sites desta natureza o Uol, o Yahoo, o Bol, o MSN, dentre outros, que disponibilizam notícias além de outros serviços, o que os torna organizações de eservice.

Desde o advento da internet, sites como estes tornam serviços acessíveis aos usuários, em estratégias variadas, buscando gerar usuários fiéis, que recomendem os serviços do site e que continuem como clientes, o que gera maior visibilidade ao fornecedor dos serviços e mais oportunidade de ou vender serviços aos clientes ou vender espaços de publicidade. A concorrência tende a ser maior na medida em que há uma expansão na demanda, o que decorre tanto da ampliação do uso da internet, quanto da disposição crescente das pessoas para usarem a internet como fonte de informação, em complemento ou em substituição a outras mídias clássicas (como rádio, televisão e jornais).

$\mathrm{Na}$ literatura de marketing e comportamento do consumidor, a lealdade é um dos principais construtos do paradigma relacional de mercado. São muitos os estudos desenvolvidos para analisar quais fatores constroem a lealdade. Dos fatores mais recorrentes, a satisfação e a confiança são os mais realçados, como será mostrado no item 2 deste artigo. Por outro lado, estes construtos também recebem influência de outros aspectos e características do próprio fornecedor dos serviços. Por análises empíricas já desenvolvidas, há indícios de que características de inovação e de qualidade são fatores efetivos de influência na satisfação e confiança. 
O objetivo do estudo é, portanto, analisar a relação entre estes construtos em uma perspectiva integrada e simultânea, ou seja, serão analisadas conjuntamente as interações e influências das características de inovação e qualidade de sites de eservice de notícias sobre a confiança e a satisfação do consumidor usuário, e as influências destas últimas (confiança e satisfação) na formação da lealdade do cliente. Os resultados poderão ser de interesse de gestores do setor de e-service para aperfeiçoamento de suas ações de marketing.

No desenvolvimento subsequente, tem-se inicialmente a discussão teórica, com a proposição do modelo de hipóteses. Em seguida, têm-se as informações sobre os procedimentos e decisões do trabalho de campo. Na sequência, apresentam-se os resultados do trabalho de campo; ao final, é feita uma síntese dos resultados e analisadas suas implicações e limitações.

\section{Discussão teórica}

Neste item são apresentados os conteúdos teóricos sobre o assunto de base do artigo. Ordenadamente, têm-se, no item 2.1, elementos teóricos gerais da teoria sobre e-service, e nos subitens de 2.2.a 2.7 são apresentadas as hipóteses do modelo, devidamente justificadas e explicadas.

\subsection{E-service}

De acordo com Ruyter, Wetzels e Kleijnen (2001), e-service é um serviço interativo centrado no conteúdo e baseado na internet, dirigido pelo consumidor e integrado com processos e tecnologias organizacionais de suporte ao consumidor, com o objetivo de fortalecer a relação entre o consumidor e o provedor do serviço. Enquanto alternativa de serviços, o e-service envolve os sites de conteúdo, nos quais a troca é executada entre o leitor e a entidade jornalística, por meio da oferta de informação.

O serviço eletrônico pode ser entendido como um dos elementos determinantes para o sucesso dos negócios em geral, por sua complementação no conceito mais amplo, o e-business (Carlson \& O'Cass, 2010), uma vez que as organizações buscam uma atuação baseada cada vez menos na redução de custos 
através da automação e no aumento da eficiência. Neste sentido, o e-service funciona como um meio de melhorar a execução das atividades empresariais e de construir relações rentáveis com os consumidores (Rust \& Kannan, 2003). A natureza da tecnologia do e-service traz particularidades que beneficiam os consumidores, como a oferta de mais informação e a possibilidade de autosserviço; isto configura a natureza experiencial do e-service (Rowley, 2006).

Portanto, é relevante que sejam analisados os fatores de influência na percepção ou comportamento do consumidor em relação ao e-service, de maneira a promover melhorias no entendimento da relação consumidor-provedor. São comuns os estudos (e.g. Chiou \& Droge, 2006; Chen \& Dibb, 2010) que buscam avaliar os fatores de impacto nos comportamentos e percepções do consumidor sobre o $e$ service, sendo destacados aqueles relativos a fatores relacionais clássicos (como qualidade, confiança e lealdade).

Nesta pesquisa são avaliadas duas características centrais do e-service de conteúdo de informações, que são a inovação e sua qualidade, em conjunto com a satisfação do consumidor, sua confiança e sua lealdade (observada nas recomendações dos sites a outros sujeitos). Com base em alguns estudos já desenvolvidos (e.g. O'Cass \& Carlson, 2012; Chang, Wang \& Yang, 2009), foi determinado um conjunto de hipóteses que identifica as relações entre estes construtos no contexto do e-service de sites de notícias.

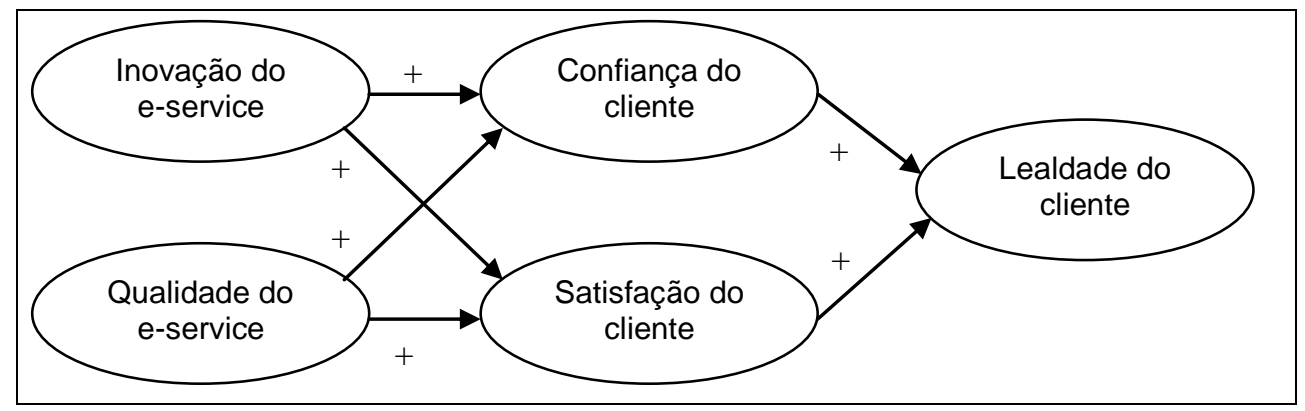

Figura 1

Modelo de hipóteses

Fonte: Elaborada pelos autores (2012) fundamentadas.

O modelo está apresentado na Figura 1, e a seguir as hipóteses são

\subsection{Inovação do e-service e confiança}

Levando em conta a meta de competitividade, é indicado que os serviços 
sejam percebidos pelos consumidores como inovadores, o que impacta de maneira positiva na credibilidade da empresa, tornando-a mais atrativa e confiável (Aaker, 2007). Em outras palavras, a inovação é uma forma de promover maior confiança do consumidor em relação à organização e suas ofertas.

No ambiente virtual, conforme afirmam Gefen, Karahanna e Straub (2003) não existem interações pessoais que estabeleçam uma relação de confiança entre a organização e o consumidor, haja vista que o site é a interface primária com a qual o consumidor tem contato. Nesse sentido, a inovação do e-service pode ser percebida em termos de apelo visual, funcionalidade, layout, informações de produtos e eficiência das transações (O'Cass \& Carlson, 2012), por meio de uma interface mais atrativa para o consumidor. Tremblay, St-Onge, Ouellet e Senecal (2009) propõem ainda que a inovação seja definida, neste contexto, pela frequência com a qual um website introduz e atualiza novas características que beneficiam o consumidor.

Desse modo, são observados alguns comportamentos intencionais relacionados com a confiança no e-service, que incluem o compartilhamento de informações pessoais, compra ou repetição de compras, ou ação com base na informação obtida no site (Luarn \& Lin, 2003). Morgan e Hunt (1994) avaliam que quando o consumidor percebe segurança e integridade na relação de troca a confiança se estabelece. Em uma perspectiva contrária, Hoffman et al. (1999) expõem que a confiança pode ser prejudicada quando o website não impõe segurança de que as informações pessoais do consumidor estão sendo preservadas durante o processo de troca on-line.

Assim, os consumidores on-line desenvolvem percepções de confiança em relação ao website por meio de uma interface inovadora, uma vez que características inovadoras podem ser favoráveis na percepção de confiança estabelecida entre o consumidor e o website (O'Cass \& Carlson, 2012). No contexto desta pesquisa, compreende-se que quanto mais inovador for um site de notícias, tanto em termos de conteúdo quanto de interface, mais percebido como confiável ele é. Este entendimento fundamenta a primeira hipótese:

- H1: A inovação do e-service em sites de notícias influencia positivamente a confiança do consumidor.

\subsection{Inovação do e-service e satisfação do consumidor}


A satisfação do consumidor é definida por Chang, Wang e Yang (2009) como uma reação psicológica do consumidor, considerando sua experiência anterior ao comparar a performance esperada e percebida de um serviço. Assim, defende-se que a inovação do e-service pode gerar experiências positivas, pois as características inovadoras do website são percebidas como valorosas pelo consumidor.

Conforme Carlson e O'Cass (2010) sugerem, a satisfação do consumidor provavelmente está relacionada às características do e-service, o que significa que, quanto mais atrativa for a interface do site, mais o consumidor deverá sentir-se satisfeito ao acessá-lo. De fato, a satisfação do consumidor reflete-se no sentimento geral que resulta do valor percebido acerca do website durante as transações online, o que tem associação com a inovação permanente como componente de benefício do fornecedor ao consumidor (Luo \& Lee, 2011).

A inovação do e-service resultará, portanto, em uma melhor avaliação geral pelo consumidor do serviço, avaliação esta que é tão melhor quanto mais inovador for o site em termos de modernidade, dinamismo e unicidade (O'Cass \& Carlson, 2012). Este entendimento permite enunciar a seguinte hipótese:

- H2: A inovação do e-service em sites de notícias influencia positivamente a satisfação do consumidor.

\subsection{Qualidade do e-service e confiança do consumidor}

A qualidade do e-service pode ser entendida como a habilidade de um website em oferecer, de maneira eficiente, o processo de compra e entrega (Zeithaml, Parasuraman \& Malhotra, 2002). Neste contexto, a confiança demonstra ser elemento essencial para a construção do relacionamento com o consumidor (O'Cass \& Carlson, 2012), ao promover a estabilidade das relações entre o consumidor e a entidade ofertante, haja vista as incertezas enfrentadas pelos indivíduos na realização das transações on-line. Conforme afirma Vieira (2010), a qualidade do serviço em geral é analisada quando o cliente compara o desempenho real com o esperado. Considerando que a confiança se constrói na medida em que o website disponibiliza informações precisas sobre suas atividades, é fácil ver a necessidade de adotar um alto padrão de qualidade na disponibilização dos serviços on-line. 
Ademais, para Henning-Thurau, Gwinner e Gremler (2002) uma sequência de boas experiências pode gerar expectativas positivas para o e-service, contribuindo tanto para o desenvolvimento da confiança quanto para a manutenção da superioridade do serviço. Diante de tais colocações, temos a seguinte hipótese:

- H3: A qualidade do e-service em sites de notícias influencia positivamente a confiança do consumidor.

\subsection{Qualidade do e-service e satisfação do consumidor}

A qualidade do serviço remete aos julgamentos dos consumidores acerca das ofertas percebidas como as melhores opções perante o mercado. Nestes termos, parece evidente a relação entre a percepção de qualidade e a satisfação do consumidor com o e-service. De acordo com Akbar e Parvez (2009), a satisfação é alcançada quando há uma avaliação positiva dos aspectos que caracterizam a relação entre as partes envolvidas na troca; portanto, entende-se que, no ambiente dos sites de notícias, a satisfação dos consumidores é influenciada pelo que ficou definido como qualidade do site.

A conclusão a que se chega é a de que, ao obter um maior nível de qualidade na transação, principalmente no que se refere à capacidade de entregar informações com agilidade, os consumidores tendem a perceber suas experiências de modo mais positivo no e-service, elevando assim sua satisfação (Carlson \& O'Cass, 2010). Isto permite enunciar a seguinte hipótese:

- H4: A qualidade do e-service em sites de notícias influencia positivamente a satisfação do consumidor.

\subsection{Confiança e lealdade do consumidor}

A lealdade do consumidor tem seu conceito aproximado à noção de comprometimento, que seria a crença do parceiro de troca de que a relação com o outro é tão importante ao ponto de justificar os esforços para mantê-la (Morgan \& Hunt, 1994). Portanto, a lealdade não pode ser entendida apenas como um comportamento de compra repetitivo (lealdade comportamental), havendo também uma lealdade de base atitudinal, que está associada ao nível de comprometimento 
do consumidor em relação a uma marca, produto ou empresa (Chiou \& Droge, 2006).

É possível verificar que a lealdade do consumidor é um resultado direto da confiança que se estabelece durante trocas de e-service, de modo que, quanto mais o consumidor se sente seguro, maior sua predisposição para desenvolver uma relação de comprometimento com o website de sua confiança. Luarn e Lin (2003) enfatizam isto, quando indicam que e-services confiáveis devem ser utilizados com mais frequência e devem provocar um maior grau de comprometimento atitudinal. Cyr (2008) também afirma que a confiança é fundamental para desenvolver a lealdade do consumidor no contexto do e-service. Tal perspectiva indica a hipótese seguinte:

H5: A confiança do consumidor em sites de notícias influencia positivamente a lealdade do consumidor.

\subsection{Satisfação e lealdade do consumidor}

No contexto on-line, a satisfação do consumidor pode ter como consequência a realização de transações repetidas em termos de uso do mesmo e-service. A perspectiva de transações repetidas indica que a satisfação do consumidor tem relação direta com sua lealdade (Chang, Wang \& Yang, 2009), ou seja, uma vez satisfeito, o consumidor tende a ser leal ao serviço, utilizando-o recorrentemente a ponto de não intencionar mudar de website.

Esta relação foi já demonstrada por Chiou e Droge (2006) na sua avaliação das intenções de lealdade, com a indicação de que um consumidor insatisfeito revela maior probabilidade de procurar canais alternativos de informação e ceder a investidas da concorrência do que um consumidor satisfeito (Anderson \& Srinivasan, 2003). Posto isto, propõe-se a seguinte hipótese:

H6: A satisfação do consumidor influencia positivamente a confiança do consumidor.

\section{Método}

Conforme indicado no item 2.2, o estudo teve por intenção avaliar um conjunto de hipóteses envolvendo construtos. Como os construtos já possuem uma larga tradição de pesquisas na literatura especializada, é possível então o teste 
quantitativo das hipóteses, por meio de técnicas estatísticas apropriadas. Assim, na construção do trabalho empírico, as decisões centrais foram relativas à mensuração dos construtos, ao design do trabalho de campo e às técnicas e ferramentas de análise. A seguir estas decisões estão descritas.

Concernente à mensuração, optou-se pela utilização de escalas previamente utilizadas em outros estudos de interesse convergentes ao deste artigo. Assim, para mensuração de inovação no site, foram utilizados três itens oriundos do estudo de O'Cass e Carlson (2012). Já para medir qualidade, foi utilizado um item do estudo de O'Cass e Carlson (2012) e mais quatro itens do estudo de Chang, Wang e Yang (2009), que ainda subsidiou os quatro itens empregados para medir confiança. A mensuração do construto satisfação foi feita com base em cinco itens procedentes do estudo de Carlson e O'Cass (2010). Por fim, para mensurar o construto lealdade, foram selecionados cinco itens do estudo de Chang, Wang e Yang (2009), juntamente com quatro itens do estudo de O'Cass e Carlson (2012). Com exceção do construto qualidade, em todos os demais a escala de verificação de concordância foi a de Likert de 10 pontos (de 1 a 10). Especificamente para qualidade, foi utilizada uma escala de verificação de 10 pontos, variando, para cada item de mensuração, de 1 (muito ruim) a 10 (muito bom). Todos os itens podem ser consultados no anexo deste artigo.

A mensuração foi feita por meio de um questionário eletrônico, disponibilizado na plataforma google docs. No instrumento, além das questões de mensuração dos construtos, foram ainda acrescentadas questões relacionadas à caracterização sócio demográfica dos respondentes, além de informações gerais sobre hábitos de uso dos sites de referência da pesquisa.

Após consolidado o questionário, foi criado um link que foi enviado a um total aproximado de 500 e-mails, além de ter sido também postado nas páginas das redes sociais dos autores. Depois de uma semana de aguardo de respostas, uma nova rodada de e-mails foi enviada. Assim, em duas semanas, foi levantado um total de 239 questionários respondidos. Considerando a adequação do tamanho da amostra para efeito de análise das hipóteses enunciadas, e considerando ainda que novas respostas ao questionário não estavam mais emergindo, a coleta foi suspensa após ficar quinze dias disponível na internet.

Os dados coletados foram primeiramente transferidos da planilha do 
google.docs para o software SPSS, para serem preparados para análise. Pelos procedimentos de análise de valores extremos e respostas evidentemente impróprias (por exemplo, todas as respostas iguais), foram excluídos 16 questionários, restando válidos para análise um total de 223 respondentes. Pelas respostas, observou-se que a maioria dos respondentes (73,5\%) estava trabalhando, ou integralmente $(51,6 \%)$ ou parcialmente $(22 \%)$; houve uma boa distribuição de gêneros (48,4\% de mulheres e $51,6 \%$ e homens); parte dos respondentes possuía somente curso superior, completo $(9,4 \%)$ ou incompleto $(32,2 \%)$, e a grande maioria possuía nível de pós-graduação $(52,9 \%)$. A renda familiar mensal ocorreu acima de $\mathrm{R} \$ 3000,00(59,5 \%)$, e a idade predominante foi entre 20 e 30 anos (com 46,2\%). Esses dados indicam uma boa variabilidade nas características dos respondentes e sugere boa adequação da amostra para os procedimentos de análise.

$\mathrm{Na}$ análise dos resultados, foi adotada a técnica de modelagem de equações estruturais com estimação de parâmetros, por meio do método de mínimos quadrados parciais. Este método tem a vantagem (frente ao método corrente de máxima verossimilhança) de não ser sensível a violações de normalidade das variáveis. Como etapa preparatória da modelagem, procedeu-se, ainda, à análise da consistência psicométrica das escalas, por meio das técnicas de análise fatorial e análise de confiabilidade. Como ferramentas de operacionalização, foram usados os softwares SPSS e WarpPLS (para modelagem de equações estruturais). Os detalhes operacionais e critérios de decisão estão indicados nas seções em que as técnicas foram aplicadas. Todos os procedimentos foram realizados com base na literatura especializada de escalas de mensuração e análise de dados (Costa, 2011; Hair et al. 2009; Lattin, Carrol, \& Green, 2011; Kock, 2012).

\section{Resultados}

Nesta seção são apresentados os resultados do estudo empírico. Ordenadamente, tem-se, no item 4.1, a análise de consistência psicométrica das escalas, um passo necessário para assegurar condições adequadas para o teste das hipóteses. No subitem 4.2 estão expostos os resultados do modelo estrutural, inclusive com a reafirmação da consistência do modelo de mensuração. No último subitem, 4.3, os resultados são discutidos. 


\subsection{Análise da consistência psicométrica das escalas}

Para testar a consistência psicométrica das escalas, foram adotados os procedimentos convencionais de análise, quais sejam: a análise fatorial exploratória, para verificação preliminar da estrutura fatorial e da dimensionalidade de cada conjunto de itens utilizado para mensurar os construtos; e a análise de consistência interna, por meio do coeficiente alpha de Cronbach. Em cada construto, ordenadamente, foram analisados: a variância extraída de cada construto (desejável ser maior que 0,5 em cada construto), os escores fatoriais (desejável que sejam maiores que 0,6 ), e o alpha (desejável que seja maior que 0,6 ).

Das verificações, somente houve necessidade de ajustes no construto lealdade, pois, das 8 variáveis inicialmente submetidas à análise fatorial, três apresentaram baixos escores. Isso obrigou a retirada de 3 variáveis, ajustando a medida (pela verificação dos conteúdos, a exclusão das variáveis não provocou alterações na validade de conteúdo das escalas). Nestes termos, optou-se pela agregação dos itens para geração de uma medida geral da variável. Deste modo, para todos os respondentes, foi adotada, como método de agregação, a extração da média dos escores marcados por item ponderado pelo respectivo escore fatorial. Este método tem a vantagem de manter a escala dentro do intervalo original de captação (de 1 a 10), além de levar em conta o nível de associação do item com o fator latente.

Os resultados destas extrações estão indicados na Tabela 1, a partir da qual é possível observar que, em todos os construtos, há uma boa consistência psicométrica, tanto em termos de resultados da análise fatorial quanto de confiabilidade. Pelas medidas de média, e considerando a escala de 1 a 10, é possível observar que as médias ficaram sempre em um nível moderado, e com um nível razoável de variabilidade (indicado pelo desvio padrão) intermediário. Em geral, isto indica boa adequação para as análises posteriores. 
Tabela 1

Resultados das extrações

\begin{tabular}{l|l|l|l|l|l}
\hline Construto & Var. ext. & $\begin{array}{l}\text { Esc. } \\
\text { mín }\end{array}$ & Alpha & Média & Desvio \\
\hline Inovação do site & 0,768 & 0,836 & 0,849 & 6,98 & 1,63 \\
Qualidade percebida & 0,683 & 0,747 & 0,861 & 7,41 & 1,30 \\
Confiança no fornecedor & 0,635 & 0,771 & 0,801 & 7,00 & 1,63 \\
Satisfação com o fornecedor & 0,659 & 0,788 & 0,870 & 7,95 & 1,33 \\
Lealdade do cliente usuário & 0,793 & 0,860 & 0,934 & 7,71 & 1,64 \\
\hline
\end{tabular}

Fonte: Dados da pesquisa (2012)

\subsection{Teste das hipóteses}

Após a consolidação da análise de consistência psicométrica das escalas dos construtos, os dados foram submetidos ao teste, conforme as indicações do software WarpPLS. Os resultados extraídos estão indicados na Tabela 2, e, na avaliação dos resultados, primeiramente verificaram-se os coeficientes betas, que são as medidas padronizadas de influência linear entre os construtos indicados.

O valor de beta é uma estimativa da influência linear, e, nesta condição (de estimativa), tem um erro associado. Deste modo, mesmo que o valor estimado seja matematicamente não nulo, estatisticamente o valor pode ser nulo. Esta nulidade é verificada pela distribuição amostral da razão entre o valor estimado e o erro padrão (que segue a distribuição $t$ de Student), e a nulidade é testada pelo $p$-valor. $O$ ponto de corte de não nulidade mais corrente é 0,05 , de tal modo que, se o $p$-valor for menor que este ponto de corte, atesta-se que o beta é não nulo, e isto sugere influência. Os valores padronizados de beta são interpretados de acordo com seu sinal, de maneira que o sinal negativo indica influência linear negativa e o sinal positivo indica influência linear positiva. Assim, desde que não nulo, quanto maior o valor de beta, maior a força da influência (positiva ou negativa) entre os construtos.

\section{Tabela 2}

Teste das hipóteses

\begin{tabular}{l|l|l|l|l}
\hline Hipótese & $\begin{array}{l}\text { Relação de influência (todas } \\
\text { positivas) }\end{array}$ & Beta & p-valor & Resultado \\
\hline H1 & Inovação para confiança & 0,199 & 0,024 & Confirmada \\
H2 & Inovação para satisfação & 0,231 & 0,000 & Confirmada \\
H3 & Qualidade para confiança & 0,492 & 0,000 & Confirmada \\
H4 & Qualidade para satisfação & 0,586 & 0,000 & Confirmada \\
H5 & Confiança para lealdade & 0,069 & 0,182 & Refutada \\
H6 & Satisfação para lealdade & 0,783 & 0,000 & Confirmada \\
\hline
\end{tabular}

Fonte: Dados da pesquisa (2012) 
Pelos resultados, é possível observar que cinco das seis hipóteses alcançaram a confirmação, tendo havido refutação somente da hipótese que previa a relação de influência linear positiva entre a confiança e a lealdade dos clientes usuários de softwares. Ou seja, a inovação se mostrou como fator de previsão consistente da confiança $(\beta=0,199, p<0,05)$ e da satisfação $(\beta=0,231, p<0,001)$. A qualidade percebida no site também se mostrou como fator de influência positiva tanto sobre a confiança $(\beta=0,492, p<0,001)$ como sobre a satisfação dos clientes usuários $(\beta=0,586, p<0,001)$. A satisfação se mostrou como um fator consistente de influência sobre a lealdade $(\beta=0,783, p<0,001)$, porém a influência esperada da confiança na lealdade não se confirmou $(\beta=0,069, p<0,182)$.

A negação da hipótese de influência positiva da confiança sobre a lealdade causa estranhamento, pois é uma relação com boa base na literatura, e que tem um forte apelo de bom senso. Para explorar mais este resultado, foi verificada a correlação amostral de Pearson, tendo-se verificado um valor de correlação entre confiança e lealdade em torno de 0,6, tanto pelas medidas compostas no SPSS $(r=0,595 p<0,001)$ quanto pela extração por meio dos construtos latentes do WarpPLS $(r=0,609 p<0,001)$. A sinalização é de que há efetivamente relacionamento linear (e, portanto, influência) entre confiança e lealdade, porém na extração simultânea com os demais construtos, esta influência é dissipada, perdendo força a ponto de se tornar marginal ou mesmo se anular.

Os resultados das hipóteses serão analisados no item 4.3, mas antes é conveniente indicar outros resultados gerados no WarpPLS que consolidam a consistência destes resultados, relativos aos coeficientes de determinação dos construtos endógenos e às evidências adicionais de consistência psicométrica.

Primeiramente, em relação ao percentual da variação explicada em cada construto endógeno do modelo, foi possível observar pelo coeficiente de determinação $\left(R^{2}\right)$ que um percentual elevado da variação do construto lealdade é explicado $\left(R^{2}=0,692\right)$ no modelo, o que é um indicador positivo para o modelo em si. Adicionalmente, o percentual de variação da satisfação explicado pelos construtos qualidade e inovação também pode ser considerado elevado $\left(R^{2}=0,590\right)$. Já o construto confiança teve uma menor porção de sua variação explicada no modelo $\left(R^{2}=0,421\right)$, o que indica que outros fatores que não estão no modelo, diferentes de 
qualidade e inovação, explicam a maior parte da variação deste construto.

Já em relação às evidências adicionais de consistência psicométrica, são destacados os resultados da variância extraída, da confiabilidade composta e do teste dos coeficientes fatoriais:

- A variância extraída no método de estimação do software (mínimos quadrados parciais) é muito próxima daquela verificada na extração por componentes principais (inovação - 0,769; qualidade $-0,658$; confiança - 0,635; satisfação $0,660$; lealdade $-0,794)$, o que reafirma a boa adequação da estrutura fatorial.

- Há ainda a confiabilidade composta, que é um indicador complementar ao alpha de Cronbach, e é entendido como adequado se for maior que 0,7 ; nos construtos da pesquisa, os valores foram sempre acima deste ponto de referência (inovação - 0,909; qualidade $-0,906$; confiança $-0,874$; satisfação $-0,906$; lealdade -0,951).

- Por fim, o software realiza ainda o teste de hipóteses da nulidade dos escores fatoriais, segundo o modelo de estimação adotado, e segundo as regras do teste aplicado, sendo possível observar que todos os escores dos fatores de cada construto foram estatisticamente não nulos.

Nestes termos, estes dados adicionais (de variância extraída e confiabilidade composta) reafirmam que os construtos foram adequadamente medidos, o que fortalece a confiança nos resultados das hipóteses acima narrados.

\subsection{Discussão dos resultados}

Em uma síntese dos resultados, observou-se uma relação significativa entre o caráter inovador do site de notícias com a confiança (resultado 1) e a satisfação (resultado 2) dos consumidores; verificou-se ainda que a qualidade do site também influencia a confiança (resultado 3) e a satisfação (resultado 4). Por fim, verificou-se que a relação entre a lealdade e a confiança no site não se comprovou (resultado 5), mas a satisfação do consumidor foi reafirmada como antecedente da lealdade (resultado 6).

Pelo primeiro resultado (a inovação do site influencia positivamente a confiança do consumidor - hipótese H1), a sinalização é de que quanto mais atrativa e confiável a interface do site, mais provável de serem estabelecidas relações de 
troca nas quais o consumidor perceba segurança e integridade. Este resultado é coerente com a literatura mais geral associada aos construtos (Hunt \& Morgan, 1994), assim como com a literatura mais recente e específica do ambiente de internet (O'Cass \& Carlson, 2012).

O segundo resultado (de que inovação também influencia positivamente a satisfação do consumidor - hipótese H2) mostra que o valor de inovação percebido no e-service conduz ao maior nível de satisfação dos usuários, o que, inclusive, já foi sinalizado em outros estudos (Luo \& Lee, 2011). Estes resultados mostram a importância de os sites atualizarem seu conteúdo e renovarem suas características, mantendo-se constantemente dinâmicos, modernos e diferenciais, o que é próprio do ambiente de internet, inovador em sua própria natureza.

Com isto, o cliente usuário será satisfeito e terá confiança de que os sites são capazes de atender às suas demandas por meio da inovação continuada, em um processo sempre voltado para se adequar às suas necessidades. Pelo resultado, a empresa terá maiores perspectivas, pela inovação, de mantê-lo confiante de que o site propaga informações precisas e atuais, elevando sua satisfação de forma direta, uma vez que, ao perceber a inovação do e-service, o consumidor está mais propenso a sentir-se satisfeito com características atrativas.

$O$ terceiro resultado (a confiança também é influenciada pela qualidade do site - hipótese $\mathrm{H} 3$ ) assegura que quanto melhor o site, mais confiável ele deverá ser, o que minimiza as incertezas inerentes às transações on-line. Sendo assim, é seguro entender que boas experiências vivenciadas durante os processos de troca geram tanto confiança quanto manutenção da superioridade do serviço (Hennigthurau et al., 2002). De fato, é razoável pensar que, ao compreender que as informações obtidas no site são corretas e atuais, o cliente usuário tende a estabelecer maior confiança com o e-service, o que pode ser melhorado na medida em que o consumidor entende que o conteúdo, o acesso e a eficiência das transações são de alta qualidade.

Adicionalmente, o quarto resultado assegurou que, quanto maior a qualidade do site observada por meio de um processo de entrega da informação de maneira eficiente (Zeithaml, Parasuraman \& Malhotra, 2002), mais satisfeito o usuário se sentirá ao acessá-lo (hipótese H4). Com isto, há mais uma evidência, agora no contexto particular do e-service, sobre uma relação já conhecida, qual seja, a de 
que, caso perceba uma maior qualidade (no site), o cliente usuário se sentirá mais satisfeito ao utilizar o serviço, por compreender que suas necessidades são atendidas, principalmente em termos de fácil e rápido acesso às informações desejadas, de maneira eficiente.

Pelo quinto resultado, ficou indicado que a confiança não é um antecedente da lealdade (hipótese H5), embora fosse possível acreditar que a lealdade do consumidor seria um resultado direto da confiança que se estabelece durante trocas de e-service. Curiosamente, este dado contraria a literatura, que em geral compreende que esta relação ocorre de maneira positiva (e.g. Reichheld \& Schefter, 2000; Huang, 2008). Assim, este estudo fica como uma referência para outros estudos associados ao assunto.

Como sugestões preliminares de explicação, os autores entendem ser provável que o usuário de sites de notícias reconheça que, ao menos em termos de conteúdo, não há maiores variações entre os diferentes provedores (inclusive porque as notícias são muito repetidas entre os sites). Além disso, a própria característica dos serviços on-line do e-service, de acesso rápido e sem barreiras físicas, permite trocar de fornecedor instantaneamente, pelo mínimo desconforto e, mais do que nunca, de forma segura quanto a invasões ou usos inapropriados de informações dos clientes.

Pelo sexto resultado, ficou evidente que quanto mais satisfeito o usuário, mais leal ele será (hipótese 6) e, provavelmente, utilizará sempre o site recorrentemente como primeira opção, sem que haja intenção de trocá-lo por outro provedor de notícias. Além disso, é provável que o consumidor recomende o site de sua preferência para outras pessoas. Este resultado, na verdade, somente reafirma uma relação já conhecida (Anderson \& Srinivasan, 2003), agora havendo uma evidência adicional oriunda do contexto particular do e-service.

Em suma, as evidências são de que a inovação e a qualidade do site são fatores evidentemente relevantes na geração de satisfação e de confiança do cliente usuário, e que a satisfação é um determinante seguro de sua lealdade. Este resultado, portanto, deve servir como balizador para que os provedores busquem manter os consumidores satisfeitos, principalmente por meio de uma interface inovadora e de um conteúdo de qualidade, como forma de elevar o nível de satisfação e de confiança dos consumidores.

De um ponto de vista teórico, além da reafirmação das hipóteses conhecidas, 
chamou atenção a mudança na relação, ao menos aqui não identificada, entre confiança e lealdade. Este resultado, que contraria uma expectativa bem fundamentada na literatura, pode ser referência para outra percepção desta relação; naturalmente, isto requer novos estudos, inclusive de natureza qualitativa, para aprofundar possíveis explicações das variações ocorridas.

\section{Considerações finais}

Este estudo teve como foco o contexto dos sites de notícias, tendo em vista o imperativo de mercado em sua demanda de celeridade de renovação do conteúdo das informações disponibilizadas pela entidade jornalística. Acredita-se que é necessário aos portais elaborar ofertas de serviços que atendam às necessidades dos usuários, gerando satisfação, confiança e lealdade no uso, sendo necessários estudos como este para efeito de aperfeiçoamento das ações de marketing das empresas. Neste sentido, a motivação e o resultado do estudo estão bem alinhados.

Com base na análise da literatura e em um levantamento empírico, com dados analisados dentro de uma abordagem quantitativa, foram obtidos resultados indicando a influência da inovação e da qualidade do site na satisfação e na confiança dos sujeitos respondentes. Mostrou-se, ainda, que estes fatores (inovação e qualidade) influenciam a lealdade, com intermediação específica da satisfação. Por outro lado, a intermediação com a confiança não se manteve coerente com os estudos realizados fora do contexto de internet, pois, embora a lealdade seja fundamental para as empresas físicas, os usuários dos sites de notícias parecem não atuar sob a lógica de influência da maior confiança em uma maior lealdade ao provedor do serviço.

De maneira geral, este artigo favoreceu um melhor entendimento do consumo de serviços de sites de notícias, ao buscar a análise de aspectos que intermediam a visitação dos usuários e a tomada de decisão no uso específico de um site. Neste sentido, tal estudo pode ser referência para outras pesquisas que tratem do consumo de notícias no ambiente virtual.

Por outro lado, o foco em sites de notícias se mostra como uma limitação, uma vez que os resultados têm restrição de explicação de outros contextos virtuais igualmente relevantes. Logo, sugere-se a realização de pesquisas que busquem 
investigar os diferentes segmentos de provedores de e-service. Adicionalmente, ficou evidenciado que as análises estatísticas empregadas não são suficientes para uma compreensão plena das relações analisadas, sendo recomendadas pesquisas qualitativas que aprofundem o entendimento sobre o consumo de notícias no ambiente virtual.

\section{Referências}

Aaker, D. (2007). Innovation: Brand it or lose it. California Management Review, $50(1), 8$.

Akbar M., \& Parvez N. (2009). Impact of Service Quality, Trust, and Customer Satisfaction on Customers Loyalty. ABAC Journal, 29(1).

Anderson R, \& Srinivasan, S. (2003). E-Satisfaction and E-Loyalty: A Contingency Framework. Psychology \& Marketing, 20(2), 123-138.

Carlson, J., \& O'Cass, A. (2010). Exploring the relationships between e-service quality, satisfaction, attitudes and behaviours in content-driven e-service web sites. Journal of services marketing, 24(2), 112-127.

Chang, H. H., Wang, Y. H., \& Yang, W. Y. (2009). The impact of e-service quality, customer satisfaction and loyalty on e-marketing: Moderating effect of perceived value. Total Quality Management, 20(4), 423-443.

Chen, J., \& Dibb, S. (2010). Consumer trust in the online retail context: Exploring the antecedents and consequences. Psychology \& Marketing, 27(4), 323-346.

Chiou, J. S., \& Droge, C. (2006). Service quality, trust, specific asset investment, and expertise: Direct and indirect effects in a satisfaction-loyalty framework. Journal of the Academy of Marketing Science, 34(4), 613-627.

Costa, F. J. (2011). Mensuração e desenvolvimento de escalas: aplicações em Administração. Rio de Janeiro: Ciência Moderna.

Cyr, D. (2008). Modeling web site design across cultures: relationships to trust, satisfaction, and e-loyalty. Journal of Management Information Systems, 24(4), 47-72.

Gefen, D., Karahanna, E., \& Straub, D. W. (2003). Trust and TAM in online shopping: An integrated model. MIS quarterly, 27(1), 51-90.

Hair, Jr. J., Black W. C., Babin, B. J., Anderson, R. E., \& Tatham, R.L. (2009). Análise multivariada de dados (6a ed.). Porto Alegre: Bookman.

Hoffman, D. L., Novak, T. P., \& Peralta, M. (1999). Building consumer trust online. Communications of the ACM, 42(4), 80-85.

Henning-Thurau, T., Gwinner, K. P., \& Gremler, D. D. (2002). Understanding 
relationship marketing outcomes an integration of relational benefits and relationship quality. Journal of service research, 4(3), 230-247.

Huang, L. (2008). Exploring the determinants of E-loyalty among travel agencies. The Service Industries Journal, 28(2), 239-254.

Kock, N. (2012). WarpPLS 3.0 User Manual. Laredo, Texas: ScriptWarp Systems.

Lattin, J., Carrol, J. D., \& Green, P. E. (2011). Análise de dados multivariados. São Paulo: Cengage Learning.

Luarn, P., \& Lin, H. (2003). A Customer Loyalty Model for E-Service Context. Journal of Electronic Commerce Research, 4(4), 156-167.

Luo, S. F., \& Lee, T. Z. (2011). The influence of trust and usefulness on customer perceptions of e-service quality. Social Behavior And Personality: An International Journal, 39(6), 825-837.

Morgan, R. M., \& Hunt, S. D. (1994). The Commitment-Trust Theory of Relationship Marketing. Journal of Marketing, 5(8), 20-38.

O'Cass, A., Carlson, J. (2012). An E-Retailing Assessement of Perceived WebsiteService Innovativeness: Implications for Website Quality Evaluations, Trust, Loyalty and Word of Mouth. Australasian Marketing Journal, 20, 28-36.

Reichheld, F. F., \& Schefter, P. (2000) E-loyalty. Harvard Business Review, 78(4), 105-113. Rowley, J. (2006). An analysis of the e-service literature: towards a research agenda. Internet Research, 16(3), 339-359.

Rust, R. T., \& Kannan, P.K. (2003). E-service: a new paradigm for business in the electronic environment. Communications of the ACM, 46(6), 36-42.

De Ruyter, K., Wetzels, M., \& Kleijnen, M. (2001). Customer adoption of e-service: an experimental study. International Journal of Service Industry Management, 12(2), 184-207.

Tremblay, E., St-Onge, A., Ouellet, U. J. F., \& Senecal, S. (2009). Website Innovativeness: Development and Validation of the Measure. Advances in Consumer Research, 8, 144-146.

Vieira, V. A. (2010). Mensuração da qualidade de serviço no varejo eletrônico e seu impacto sobre as intenções comportamentais. RAE-Revista de Administração de Empresas, 50(2), 199-214.

Zeithaml, V. A., Parasuraman, A., \& Malhotra, A. (2002). Service quality delivery through web sites: a critical review of extant knowledge. Journal of the academy of marketing science, 30(4), 362-375. 


\section{Apêndice - Itens finais das escalas e medidas descritivas Qualidade}

\section{Itens}

Para mim, a qualidade DO SITE de notícias é...

Média

7,73

7,24

Eu acho que a seleção de notícias no site é...

6,89

de notícias é suficientemente...

Para mim, o site de notícias tem uma organização...

Para mim, a facilidade e rapidez para encontrar uma notícia no site é

suficientemente...

\begin{tabular}{|c|c|c|}
\hline \multicolumn{3}{|l|}{ Inovação } \\
\hline Itens & Média & Desvio \\
\hline Considero o site de notícias bastante inovador & 6,94 & 1,887 \\
\hline Para mim, o site de notícias oferece um diferencial para seus leitores & 7,07 & 1,894 \\
\hline O site de notícias da minha preferência tem características bem inovadoras & 6,92 & 1,811 \\
\hline
\end{tabular}

\section{Satisfação com o site}

\begin{tabular}{l|l|l}
\hline Itens & Média & Desvio \\
\hline Eu estou satisfeito com a decisão de usar o site de notícias da minha preferência & 8,09 & 1,680 \\
Eu acho que faço o certo em utilizar o site de notícias da minha preferência & 8,48 & 1,509 \\
Considero que a decisão de usar este site de notícias foi sábia & 7,56 & 1,744 \\
O site de notícias que escolhi satisfaz as minhas necessidades & 7,57 & 1,712 \\
Eu gosto de usar este site de notícias & 8,06 & 1,564 \\
\hline
\end{tabular}

\section{Confiança}

\begin{tabular}{l|l|l}
\hline Itens & Média & Desvio \\
\hline Eu confio que minhas informações pessoais estão seguras ao visitar este site de & 6,36 & 2,385 \\
notícias & 6,90 & 1,903 \\
$\begin{array}{l}\text { Eu estou certo de que as informações comunicadas pelo site de notícias são } \\
\text { seguras }\end{array}$ & 7,29 & 2,174 \\
$\begin{array}{l}\text { Eu me sinto seguro (por exemplo, com a instalação de vírus no computador) em } \\
\text { utilizar este site de notícias }\end{array}$ & 7,44 & 1,754 \\
\hline \begin{tabular}{l} 
Eu acredito que o site de notícias da minha preferência é confiável \\
\hline
\end{tabular}
\end{tabular}

\section{Lealdade}

\begin{tabular}{l|l|l}
\hline Itens & Média & Desvio \\
\hline Quando eu preciso ver alguma notícia, este site de notícias é minha primeira & 7,95 & 1,997 \\
escolha & 7,69 & 1,879 \\
Eu encorajaria amigos e parentes a usarem este site de notícias & 7,82 & 1,820 \\
Eu acredito que este é meu site de notícias preferido & 7,73 & 1,799 \\
Eu recomendaria este site de notícias para outras pessoas que pedissem meu & & \\
conselho & 7,39 & 1,733 \\
\hline Para mim, este é o melhor site de notícias para estar bem informado
\end{tabular}

